\title{
UNIQUE SOLUTIONS FOR A CLASS OF DISCONTINUOUS DIFFERENTIAL EQUATIONS
}

\author{
ALBERTO BRESSAN
}

Abstract. This paper is concerned with the Cauchy Problem

$$
\dot{x}(t)=f(t, x(t)), \quad x\left(t_{0}\right)=x_{0} \in \mathbf{R}^{n},
$$

where the vector field $f$ may be discontinuous with respect to both variables $t, x$. If the total variation of $f$ along certain directions is locally finite, we prove the existence of a unique solution, depending continuously on the initial data.

1. Introduction. Let $f$ be a vector field on $\mathbb{R}^{n}$. By definition, a Carathéodory solution of the Cauchy Problem

$$
\dot{x}(t)=f(t, x(t)), \quad x\left(t_{0}\right)=x_{0} \in \mathbb{R}^{n},
$$

is an absolutely continuous function $t \rightarrow x(t)$ which takes the value $x_{0}$ at $t=t_{0}$ and satisfies the differential equation in (1.1) at almost every $t$. If $f$ is not continuous, Peano's theorem does not apply and (1.1) may not have any solution. Some authors have thus introduced new definitions of generalized or relaxed solutions for (1.1), for which a satisfactory existence theorem could then be proven $[\mathbf{5}, \mathbf{6}, \mathbf{9}]$. An alternative approach to discontinuous O.D.E.'s, pursued in $[\mathbf{4}, \mathbf{7}, \mathbf{8}]$, relies on the study of certain conditions on $f$ which are weaker than continuity, yet sufficient to guarantee the existence of Carathéodory solutions. This led to the investigation of directional continuity. For a fixed $M>0$, consider the cone

$$
\Gamma^{M}=\left\{(t, x) \in \mathbf{R}^{n+1} ;\|x\| \leq M t\right\} .
$$

We say that a map $f: \mathbf{R}^{n+1} \rightarrow \mathbf{R}^{n}$ is $\Gamma^{M}$-continuous if, for every $\left(t_{0}, x_{0}\right) \in \mathbf{R}^{n+1}$ and $\varepsilon>0$, there exists $\delta>0$ such that

$$
t_{0} \leq t<t_{0}+\delta, \quad\left\|x-x_{0}\right\| \leq M\left(t-t_{0}\right) \Rightarrow\left\|f(t, x)-f\left(t_{0}, x_{0}\right)\right\|<\varepsilon .
$$

Assuming that $\|f(t, x)\| \leq L<M$ for all $t, x$, solutions of O.D.E.'s with $\Gamma^{M_{-}}$ continuous right-hand sides were obtained in [7] as limits of polygonal approximations, in [2] through an application of Schauder's fixed point theorem, and in [3] by means of an upper semicontinuous, convex-valued regularization. These results acquire additional interest in connection with the theory of multivalued differential equations. Indeed, the existence of directionally continuous selections for lower semicontinuous multifunctions now provides a very effective tool for the study of differential inclusions [2, 3].

The present paper is concerned with the problem of uniqueness and continuous dependence. In the classical theory, the uniqueness of solutions of (1.1) is proved assuming that $f$ is locally Lipschitz continuous. Here we consider a much weaker

Received by the editors November 2, 1987.

1980 Mathematics Subject Classification (1985 Revision). Primary 34A10. 
condition, which does not imply the continuity of $f$. Let $\prec$ be the partial ordering on $\mathbb{R}^{n+1}$ induced by the cone $\Gamma^{M}$ :

$$
(t, x) \prec\left(t^{\prime}, x^{\prime}\right) \quad \text { iff }\left\|x^{\prime}-x\right\| \leq M\left(t^{\prime}-t\right) .
$$

Using this ordering, one can define a class of vector fields with bounded "directional variation".

DEFINITION 1. A vector field $f: \mathbb{R}^{n+1} \rightarrow \mathbb{R}^{n}$ has bounded $\Gamma^{M}$-variation if there exists a constant $C$ such that

$$
\sum_{i=1}^{N}\left\|f\left(t_{i}, x_{i}\right)-f\left(t_{i-1}, x_{i-1}\right)\right\| \leq C
$$

for every finite sequence $\left(t_{i}, x_{i}\right), i=0,1, \ldots, N$, with $\left(t_{0}, x_{0}\right) \prec\left(t_{1}, x_{1}\right) \prec \cdots \prec$ $\left(t_{N}, x_{N}\right)$.

DEFINITION 2. A vector field $f: \mathbb{R}^{n+1} \rightarrow \mathbb{R}^{n}$ has locally bounded $\Gamma^{M}$-variation if, for every $\left(t_{0}, x_{0}\right) \in \mathbb{R}^{n+1}$, there exist $\delta>0$ and a constant $C$ such that

$$
\sum_{i=1}^{N}\left\|f\left(t_{i}, x_{i}\right)-f\left(t_{i-1}, x_{i-1}\right)\right\| \leq C
$$

for every finite sequence $\left(t_{i}, x_{i}\right), i=1, \ldots, N$, satisfying

$$
\left(t_{0}, x_{0}\right) \prec\left(t_{1}, x_{1}\right) \prec \cdots \prec\left(t_{N}, x_{N}\right), \quad t_{N}<t_{0}+\delta .
$$

Our main result shows that if $f$ has locally bounded directional variation, then the solution of (1.1) is unique:

THEOREM 1. Let $f: \mathbb{R}^{n+1} \rightarrow \mathbb{R}^{n}$ be a vector field with locally bounded $\Gamma^{M}$ variation. If $\|f(t, x)\| \leq L<M$ for all $t, x$, then the Cauchy Problem (1.1) has a unique forward solution $x(\cdot)$, which is defined on $\left[t_{0}, \infty\right)$. Moreover, the restriction of $x(\cdot)$ to any bounded interval $\left[t_{0}, T\right]$ depends continuously on the initial value $x_{0}$.

In $\S 2$ we establish an intermediate result. The proof of Theorem 1 is then completed in $\S 3$; it relies on the construction of a directionally continuous version of $f$ and on a proper use of the classical Contraction Mapping Principle.

2. An auxiliary theorem. The following uniqueness result for solutions of the Cauchy Problem

$$
\dot{x}(t)=f(t, x(t)), \quad x(0)=0 \in \mathbf{R}^{n},
$$

will be instrumental for the proof of Theorem 1 .

THEOREM 2. Assume that there exist constants $L, M, \delta>0$ and a function $\phi: \Gamma^{M} \rightarrow \mathbf{R}$ such that

(i) $\|f(t, x)\| \leq L<M$,

(ii) $\lim _{t \rightarrow 0^{+},\|x\| \leq M t} \phi(t, x)=\phi(0,0)=0$,

(iii) $\|f(t, x)-f(s, y)\| \leq \phi(t, x)-\phi(s, y)$ whenever $(t, x),(s, y) \in \Gamma^{M}, 0 \leq s \leq$ $t<\delta,\|x-y\| \leq M(t-s)$.

Then (2.1) has a unique forward solution, defined on some positive interval $[0, T]$.

Proof. Using (ii), choose $T \in(0, \delta / 2)$ such that

$$
\phi(t, x) \leq(M-L) / 4
$$


whenever $0 \leq t \leq 2 T,\|x\| \leq M t$. Consider the set $\mathbf{K}$ of all continuous mappings $y:[0, T] \rightarrow \mathbb{R}^{n}$ with $y(0)=0$ and with Lipschitz constant $L$. Define the Picard operator $P: K \rightarrow K$ by setting

$$
P(y)(t)=\int_{0}^{t} f(s, y(s)) d s .
$$

For any $y \in \mathbf{K}$, (iii) and (2.2) imply that the map $s \rightarrow f(s, y(s))$ has bounded variation, hence the integral in (2.3) is well defined. Clearly $P(y) \in K$ because of (i). We claim that $P$ is a strict contraction. For any $y_{1}, y_{2} \in K$, set

$$
\sigma=\left\|y_{1}-y_{2}\right\|_{\mathscr{C} O}=\max _{t \in[0, T]}\left\|y_{1}(t)-y_{2}(t)\right\| \leq 2 L T .
$$

Call $\xi=\sigma / 2 M$ and define the auxiliary map

$$
z(t)=\left[y_{1}(t-\xi)+y_{2}(t-\xi)\right] / 2, \quad t \in[\xi, T+\xi] .
$$

Observe that $\xi \leq T$ and that $z$ has Lipschitz constant $L$. Moreover

$$
\left\|z(t+\xi)-y_{i}(t)\right\| \leq \sigma / 2=M[(t+\xi)-t],
$$

therefore we can apply (iii) and deduce

$$
\left\|f(t+\xi, z(t+\xi))-f\left(t, y_{i}(t)\right)\right\| \leq \phi(t+\xi, z(t+\xi))-\phi\left(t, y_{i}(t)\right)
$$

for all $t \in[0, T], i=1,2$. Observe that (iii) trivially implies

$$
\|x-y\| \leq M(t-s) \Rightarrow \phi(s, y) \leq \phi(t, x) \text {. }
$$

In particular, the maps $t \rightarrow \phi\left(t, y_{i}(t)\right)$ and $t \rightarrow \phi(t+\xi, z(t+\xi))$ are nondecreasing. For $0 \leq r \leq(M-L) / 4, i=1,2$, define

$$
\begin{gathered}
\tau_{i}(r)=\inf \left\{t \in[0, T] ; \phi\left(t, y_{i}(t)\right) \geq r\right\} \wedge T, \\
\tau_{z}(r)=\inf \{t \in[0, T] ; \phi(t+\xi, z(t+\xi)) \geq r\} \wedge T .
\end{gathered}
$$

We claim that

$$
0 \leq \tau_{i}(r)-\tau_{z}(r) \leq \sigma /(M-L) .
$$

Indeed, the first inequality follows from (2.6), (2.8) which imply

$$
\phi\left(t, y_{i}(t)\right) \leq \phi(t+\xi, z(t+\xi)) \quad \forall t \in[0, T] .
$$

To prove the second inequality, set $\tau=\tau_{z}(r)$. If $\tau=T$, the conclusion is obvious. Otherwise, for any $\tau^{\prime} \in(\tau, T]$, the choice of $\xi$ implies

$$
\begin{aligned}
& \left\|z\left(\tau^{\prime}+\xi\right)-y_{i}\left(\tau^{\prime}+\frac{\sigma}{M-L}\right)\right\| \\
& \quad \leq\left\|z\left(\tau^{\prime}+\xi\right)-y_{i}\left(\tau^{\prime}\right)\right\|+\left\|y_{i}\left(\tau^{\prime}\right)-y_{i}\left(\tau^{\prime}+\frac{\sigma}{M-L}\right)\right\| \\
& \quad \leq \frac{\sigma}{2}+\frac{\sigma}{M-L} \cdot L=M\left(\frac{\sigma}{M-L}-\xi\right) .
\end{aligned}
$$

Therefore (2.8) yields

$$
r \leq \phi\left(\tau^{\prime}+\xi, z\left(\tau^{\prime}+\xi\right)\right) \leq \phi\left(\tau^{\prime}+\frac{\sigma}{M-L}, y_{i}\left(\tau^{\prime}+\frac{\sigma}{M-L}\right)\right)
$$


hence $\tau_{i}(r) \leq \tau^{\prime}+\sigma /(M-L)$ for all $\tau^{\prime}>\tau$, completing the proof of (2.9). Relying on (2.2) and (2.9) we now obtain our basic estimate, through a change in the order of integration:

$$
\begin{aligned}
\int_{0}^{T} & \left\|f(t+\xi, z(t+\xi))-f\left(t, y_{i}(t)\right)\right\| d t \\
& \leq \int_{0}^{T}\left[\phi(t+\xi, z(t+\xi))-\phi\left(t, y_{i}(t)\right)\right] d t=\int_{0}^{T} \int_{\phi\left(t, y_{i}(t)\right)}^{\phi(t+\xi, z(t+\xi))} d r d t \\
& =\int_{0}^{(M-L) / 4}\left[\tau_{i}(r)-\tau_{z}(r)\right] d r \leq \frac{M-L}{4} \cdot \frac{\sigma}{M-L}=\frac{\sigma}{4}
\end{aligned}
$$

From (2.10) it follows that

$$
\int_{0}^{T}\left\|f\left(t, y_{1}(t)\right)-f\left(t, y_{2}(t)\right)\right\| d t \leq \frac{\sigma}{2}=\frac{\left\|y_{1}-y_{2}\right\|}{2} .
$$

Therefore the Picard operator $P$ is a strict contraction on $K$ and has a unique fixed point $x(\cdot)$, which yields the unique Carathéodory solution of $(2.1)$ on $[0, T]$.

3. Proof of Theorem 1. For simplicity we assume $t_{0}=0, x_{0}=0 \in \mathbf{R}^{n}$, which is not restrictive. Define the auxiliary vector field $\tilde{f}: \mathbf{R}^{n+1} \rightarrow \mathbf{R}^{n}$ by setting

$$
\tilde{f}(t, x)=\lim _{s \rightarrow t^{+}} f(s, x) .
$$

The limit in (3.1) always exists because, for $\varepsilon>0$ small enough, the map $s \rightarrow$ $f(s, x)$ has bounded variation on $[t, t+\varepsilon)$. Set $\widetilde{M}=(M+L) / 2$. For all $(t, x)$ inside the cone

$$
\Gamma^{\widetilde{M}}=\{(t, x) ;\|x\| \leq \widetilde{M} t\}
$$

define

$$
\phi(t, x)=\sup \left\{\sum_{i=1}^{N}\left\|\tilde{f}\left(t_{i}, x_{i}\right)-\tilde{f}\left(t_{i-1}, x_{i-1}\right)\right\|\right\}
$$

the supremum being taken over all finite sequences $\left\{\left(t_{i}, x_{i}\right) ; i=0, \ldots, N\right\}$ with $N \geq 1,\left(t_{0}, x_{0}\right)=(0,0),\left(t_{N}, x_{N}\right)=(t, x)$ and $\left\|x_{i}-x_{i-1}\right\| \leq \widetilde{M}\left(t_{i}-t_{i-1}\right)$ for all $i$. Some properties of $\tilde{f}$ and $\phi$ will be examined in the next lemmas.

LEMMA 1. The vector field $\tilde{f}$ is $\Gamma^{\widetilde{M}}$-continuous.

Proof. Assume, on the contrary, that there exists a sequence of points $\left(t_{n}, x_{n}\right)$ converging to $(t, x)$, with $\left\|x_{n}-x\right\| \leq \widetilde{M}\left(t_{n}-t\right)$ but

$$
\left\|\tilde{f}\left(t_{n}, x_{n}\right)-\tilde{f}(t, x)\right\|>\eta>0 \quad \forall n \geq 1 .
$$

By (3.1), we can choose $t_{n}^{\prime} \in\left(t_{n}, t_{n}+1 / n\right]$ suitably close to $t_{n}$ and still have

$$
\left\|f\left(t_{n}^{\prime}, x_{n}\right)-\tilde{f}(t, x)\right\|>\eta \quad \forall n \geq 1 \text {. }
$$

Let $\left\{s_{m}\right\}$ be a sequence strictly decreasing to $t$, with the property

$$
\left\|f\left(s_{m}, x\right)-\tilde{f}(t, x)\right\|<\eta / 2 \quad \forall m \geq 1
$$


Let $\delta>0$ be given. By induction, we now construct a sequence $\left(\tau_{j}, y_{j}\right)$ such that, recalling (1.3),

$$
\begin{gathered}
0<\tau_{j}<\delta, \quad\left(\tau_{j+1}, y_{j+1}\right) \prec\left(\tau_{j}, y_{j}\right) \forall j \geq 1, \\
\begin{cases}\text { if } j \text { is even, } & \left(\tau_{j}, y_{j}\right)=\left(t_{n_{j}}^{\prime}, x_{n_{j}}\right) \text { for some } n_{j}, \\
\text { if } j \text { is odd, } & \left(\tau_{j}, y_{j}\right)=\left(s_{m_{j}}, x\right) \quad \text { for some } m_{j} .\end{cases}
\end{gathered}
$$

This can be done as follows. First choose $m_{1}$ such that $s_{m_{1}}<\delta$ and set $\left(\tau_{1}, y_{1}\right)=$ $\left(s_{m_{1}}, x\right)$. If $\left(\tau_{j}, y_{j}\right)$ has been defined for all $j<2 k$, select $n_{2 k}$ so large that $\widetilde{M} t_{n_{2 k}}^{\prime} \leq$ $M\left(\tau_{2 k-1}-t_{n_{2 k}}^{\prime}\right)$ and set $\left(\tau_{2 k}, y_{2 k}\right)=\left(t_{n_{2 k}}^{\prime}, x_{n_{2 k}}\right)$. Then select $m_{2 k+1}$ so large that $\left\|y_{2 k}\right\|=\left\|x_{n_{2 k}}\right\| \leq M\left(\tau_{2 k}-s_{m_{2 k+1}}\right)$ and set $\left(\tau_{2 k+1}, y_{2 k+1}\right)=\left(s_{m_{2 k+1}}, x\right)$. All this is possible because the sequences $\left\{t_{n}^{\prime}\right\},\left\{s_{m}\right\}$ converge to $t$ and $\left\|x_{n}-x\right\|<M\left(t_{n}^{\prime}-t\right)$ for every $n$. The sequence $\left\{\left(\tau_{j}, y_{j}\right) ; j \geq 1\right\}$ then satisfies (3.6), (3.7). From (3.4) and (3.5) it follows that

$$
\sum_{j=1}^{\infty}\left\|f\left(\tau_{j+1}, y_{j+1}\right)-f\left(\tau_{j}, y_{j}\right)\right\|=\infty
$$

hence the vector field $f$ cannot have locally bounded $\Gamma^{M}$-variation near the point $(t, x)$. This contradiction proves the lemma.

LEMMA 2. The sets of Carathéodory solutions for the differential equations $\dot{x}(t)=f(t, x(t))$ and $\dot{x}(t)=\tilde{f}(t, x(t))$ coincide.

PROOF. Let $u:[a, b] \rightarrow \mathbf{R}^{n}$ be any continuous map with Lipschitz constant $L$. Define $J$ as the set of times $t \in[a, b]$ for which there exists some sequence $\left\{t_{k}\right\}$, strictly decreasing to $t$, with $f\left(t_{k}, u\left(t_{k}\right)\right)$ converging to $f(t, u(t))$. The measurability of the map $t \rightarrow f(t, u(t))$ implies that meas $(J)=b-a$ (see Lemma 2.3 in [3] for details). Since $L<\widetilde{M}$, the $\Gamma^{\widetilde{M}}$-continuity of $\tilde{f}$ implies $\tilde{f}(t, u(t))=f(t, u(t))$ for all $t \in J$, hence almost everywhere on $[a, b]$. Lemma 2 is now clear, because every integral curve for the vector fields $f$ or $\tilde{f}$ is Lipschitz continuous with constant $L$.

LEMMA 3. There exists $\delta>0$ such that $\phi$ is bounded on the set $\Delta=\{(t, x) ;\|x\|$ $\leq \widetilde{M} t, 0 \leq t<\delta\}$. Moreover,

$$
\|\tilde{f}(t, x)-\tilde{f}(s, y)\| \leq \phi(t, x)-\phi(s, y)
$$

for all $(t, x),(s, y) \in \Delta$ with $\|x-y\| \leq \widetilde{M}(t-s)$;

$$
\lim _{\substack{t \rightarrow 0^{+} \\\|x\| \leq \widetilde{M} t}} \phi(t, x)=0 .
$$

PROOF. We begin by proving the last assertion. If (3.10) fails, then there exists a constant $\eta>0$ such that, for every $\varepsilon>0$, one can find a finite sequence $\left\{\left(t_{i}, x_{i}\right) ; i=0, \ldots, N\right\}$ satisfying

$$
\begin{gathered}
\left(t_{0}, x_{0}\right)=(0,0), \quad t_{N}<\varepsilon, \quad\left\|x_{i}-x_{i-1}\right\| \leq \widetilde{M}\left(t_{i}-t_{i-1}\right), \\
\sum_{i=1}^{N}\left\|\tilde{f}\left(t_{i}, x_{i}\right)-\tilde{f}\left(t_{i-1}, x_{i-1}\right)\right\|>\eta
\end{gathered}
$$


Choosing $\tau_{i}>t_{i}$ suitably close to $t_{i}$, we obtain a finite sequence $\left(\tau_{i}, x_{i}\right)$ which satisfies

$$
\begin{gathered}
0<\tau_{0}<\cdots<\tau_{N}<\varepsilon, \quad\left\|x_{i}\right\| \leq \widetilde{M} \tau_{i}, \quad\left\|x_{i}-x_{i-1}\right\| \leq M\left(\tau_{i}-\tau_{i-1}\right) \\
\sum_{i=1}^{N}\left\|f\left(\tau_{i}, x_{i}\right)-f\left(\tau_{i-1}, x_{i-1}\right)\right\|>\eta
\end{gathered}
$$

Let $\delta>0$ be given. Construct a sequence of finite sets $S_{k}=\left\{\left(\tau_{i}^{k}, x_{i}^{k}\right) ; i=\right.$ $\left.0, \ldots, N_{k}\right\}, k \geq 1$, inductively as follows. Define $S_{1}$ to be any finite sequence for which (3.13) and (3.14) hold with $\varepsilon=\delta$. When $S_{k-1}$ has been defined, let $S_{k}$ be any finite sequence which satisfies (3.13) and (3.14) with $\varepsilon=\tau_{0}^{k-1}(M+\widetilde{M}) /(M-\widetilde{M})$. Since $\left\|x_{0}^{k-1}\right\| \leq \widetilde{M} \tau_{0}^{k-1},\left\|x_{N_{k}}^{k}\right\| \leq \widetilde{M} \tau_{N_{k}}^{k}$, this choice of $\varepsilon$ implies

$$
\left(\tau_{N_{k}}^{k}, x_{N_{k}}^{k}\right) \prec\left(\tau_{0}^{k-1}, x_{0}^{k-1}\right) \quad \forall k>1 .
$$

Because of (3.15), the set $S=\bigcup_{k \geq 1} S_{k}$ is totally ordered by the relation $\prec$ defined at (1.3). We can thus arrange its elements into a unique decreasing sequence, say $S=\left\{\left(\tau_{j}, x_{j}\right), j \geq 0\right\}$, with $\left(\tau_{j}, x_{j}\right) \prec\left(\tau_{j-1}, x_{j-1}\right)$ for all $j \geq 1$. Since every $S_{k}$ satisfies (3.14), it follows that

$$
\sum_{j=1}^{\infty}\left\|f\left(\tau_{j}, x_{j}\right)-f\left(\tau_{j-1}, x_{j-1}\right)\right\|=\infty .
$$

By (3.16), $f$ cannot have locally bounded $\Gamma^{M}$-variation near the point $(0,0)$. This contradiction proves (3.10). The existence of a set $\Delta$ where $\phi$ is bounded is an obvious consequence of (3.10). The "dynamic programming" equation (3.9) follows easily from the definition of $\phi$ at (3.2).

It is now possible to complete the proof of Theorem 1. By Lemma 3, one can apply Theorem 2 to the vector field $\tilde{f}$ (with $M$ replaced by $\widetilde{M}$ ) and obtain the existence of a unique local solution $x(\cdot)$ for the Cauchy Problem

$$
\dot{x}(t)=\tilde{f}(t, x(t)), \quad x\left(t_{0}\right)=x_{0},
$$

on some forward interval $\left[t_{0}, T\right]$. By Lemma $2, x(\cdot)$ is also the unique solution of $(1.1)$ on $\left[t_{0}, T\right]$. Since $f$ is bounded, this solution can be uniquely extended to $\left[t_{0}, \infty\right)$. To prove the continuous dependence on the initial data, consider the upper semicontinuous compact convex valued multifunction $F$ :

$$
F(t, x)=\bigcap_{\varepsilon>0} \overline{\operatorname{co}}\{f(s, y) ;|s-t|<\varepsilon,\|y-x\|<\varepsilon\},
$$

where $\overline{\text { co }}$ stands for closed convex hull. By Lemma 3.2 in [3], the Carathéodory solutions of (1.1) coincide with those of the multivalued Cauchy Problem

$$
\dot{x}(t) \in F(t, x(t)), \quad x\left(t_{0}\right)=x_{0} .
$$

For any fixed $T>t_{0}$, let $\mathscr{T}\left(x_{0}\right) \subseteq \mathscr{C}\left(\left[t_{0}, T\right] ; \mathbf{R}^{n}\right)$ denote the family of solutions of (3.19). The set-valued map $x_{0} \rightarrow \mathscr{T}\left(x_{0}\right)$ is then upper semicontinuous [1, p. 104]. By uniqueness, in our case $\mathscr{T}\left(x_{0}\right)$ reduces to a single element, therefore it depends continuously on $x_{0}$. 


\section{REFERENCES}

1. J. P. Aubin and A. Cellina, Differential inclusions, Springer, Berlin, 1984.

2. A. Bressan, Directionally continuous selections and differential inclusions, Funkcial. Ekvac. 31 (1988), 459-470.

3. __ Upper and lower semicontinuous differential inclusions. A unified approach, Controllability and Optimal Control (H. Sussmann, ed.), Dekker, New York (to appear).

4. A. Cambini and S. Querci, Equazioni differenziali del primo ordine con secondo membro discontinuo rispetto all'incognita, Rend. Istit. Mat. Univ. Trieste 1 (1969), 89-97.

5. A. F. Filippov, Differential equations with discontinuous right-hand sides, Trans. Amer. Math. Soc. 42 (1964), 199-231.

6. O. Hajek, Discontinuous differential equations. I, J. Differential Equations 32 (1979), 149-170.

7. A. Pucci, Sistemi di equazioni differenziali con secondo membro discontinuo rispetto all'incognita, Rend. Istit. Mat. Univ. Trieste 3 (1971), 75-80.

8. _ Traiettorie di campi di vettori discontinui, Rend. Istit. Mat. Univ. Trieste 8 (1976), 84-93.

9. R. Sentis, Equations differentielles à second membre mesurable, Boll. Un. Mat. Ital. 15 B (1978), 724-742.

Department of Mathematics, UNiversity of Colorado, Boulder, Colorado 80309 